\title{
Multi-access drug delivery network and stability
}

This article was published in the following Dove Press journal:

International Journal of Nanomedicine

22 August 201I

Number of times this article has been viewed

\section{S Mitatha}

N Moongfangklang'

MA Jalil ${ }^{2}$

N Suwanpayak ${ }^{3,4}$

$\mathrm{J} \mathrm{Ali}^{5}$

PP Yupapin ${ }^{4}$

'Hybrid Computing Research Laboratory, Faculty of Engineering, King Mongkut's Institute of

Technology Ladkrabang, Bangkok,

Thailand; ' Ibnu Sina Institute of

Fundamental Science Studies,

Nanotechnology Research Alliance,

Universiti Teknologi Malaysia (UTM),

Johor Bahru, Malaysia; ${ }^{3}$ King Mongkut's

Institute of Technology Ladkrabang,

Chumphon Campus, Chumphon,

Thailand; ${ }^{4}$ Nanoscale Science and

Engineering Research Alliance

(N'SERA), Advanced Research Center

for Photonics, Faculty of Science, King

Mongkut's Institute of Technology

Ladkrabang, Bangkok, Thailand;

${ }^{5}$ Institute of Advanced Photonics

Science, Nanotechnology Research

Alliance, Universiti Teknologi Malaysia

(UTM), Johor Bahru, Malaysia
Correspondence: PP Yupapin

Nanoscale Science and Engineering

Research Alliance (N'SERA), Advanced

Research Center for Photonics,

Faculty of Science, King Mongkut's

Institute of Technology Ladkrabang,

Bangkok 10520, Thailand

Tel +66 23298414

Fax +66 23298410

Email kypreech@kmitl.ac.th, isoqt@ kmitl.ac.th
Abstract: A novel design of a multi-drug delivery network and diagnosis using a molecular network is proposed. By using a pair of tweezers to generate the intense optical vortices within the PANDA ring resonator, the required molecules (drug volumes) can be trapped and moved dynamically within the molecular bus networks, in which the required drug delivery targets can be achieved within the network. The advantage of the proposed system is that the diagnostic method can be used within a tiny system (thin film device or circuit), which is available as an embedded device for diagnostic use in patients. In practice, the large molecular networks such as ring, star, and bus networks can be integrated to form a large drug delivery system. The channel spacing of the trapped volumes (molecules) within the bus molecular networks can be provided by using the appropriate free spectrum range, which is analyzed and discussed in the terms of crosstalk effects. In this work, crosstalk effects of about $0.1 \%$ are noted, which can be neglected and does not affect the network stability.

Keywords: drug delivery network, molecular networks, molecular diagnosis, neural system and network

\section{Introduction}

Human organs contain blood and tissue fluid. The heart pumps blood through the arteries and capillaries and returns to the heart via veins. By providing oxygen and nutrients to every cell of the body, all the cells are refreshed when molecules such as oxygen and nutrients move into tissue fluid from the blood. The blood circulation carries away waste products, ${ }^{1}$ each red blood cell $7-8.5 \mu \mathrm{m}$ in diameter passes through the narrow capillaries smaller than $3 \mu \mathrm{m}$ in diameter. Most capillaries range in diameter from 7 to $9 \mu \mathrm{m}$, and branch without changing in diameter. The circulation of blood through the human body is divided into two interlocking systems, venous and arterial. Together, they keep a dynamic interchange of blood moving to and from the heart and lungs. ${ }^{2}$ Several studies have been done to understand red blood cell transportation in the capillaries network, ${ }^{3}$ overall elasticity of the capillary system, apparent membrane viscosity, thickness of the double layer of electrical charges, adhesion of red blood cells in vascular to fabricate the same blood flow system, ${ }^{4}$ and pulmonary network via micro-fluidics system. ${ }^{5}$

Optical trapping was invented by Ashkin et al. ${ }^{6}$ It has emerged as a powerful tool with a wide range applications in biology, physics, engineering, and medicine. ${ }^{7}$ Optical trapping and manipulation of viruses, living cells, bacteria, and organelles without damage by laser radiation have been demonstrated. ${ }^{8-10}$ In medicine and the application of nanotechnology, a single red blood cell (RBC) deformability test has been performed by 
optical trapping plastic in microfluidics chip ${ }^{3,11}$ and labon-a-chip for RBC transportation in capillary network to circulate oxygen and carbon dioxide throughout the human body. ${ }^{12}$ Optical trapping for manipulation of molecules in liquid core capillaries and its application to drug delivery has been reported by Suwanpayak et al, ${ }^{13}$ who used a PANDA ring resonator to form, transmit, and receive the microscopic volume (of drug) by controlling the ring parameters. A microscopic volume can be trapped and moved dynamically within the wavelength router or network.

An optical buffer is recognized as an essential component in a wavelength router and network, in which the data packets can be stored to resolve the packet contention problem and also delay the outgoing packets. ${ }^{14,15}$ In this case the packets of tweezers (trapped drug volumes) are transported through the network in a similar manner. In practice, the optical routers are useful for various applications, especially for molecular networks. ${ }^{16-18}$ Recently, promising techniques of microscopic volume trapping and transport within the add/drop multiplexer have been reported theoretically ${ }^{19}$ and experimentally, ${ }^{20}$ in which the transporter is an optical tweezer. The static tweezers are now well recognized and used. The optical tweezer generation technique has become a powerful tool for manipulation of micrometer-sized particles. Dynamic tweezers are now also used in practical work. ${ }^{21-23}$ Schulz et al have shown that the transfer of trapped atoms between two optical potentials could be performed. ${ }^{24}$ The optical tweezers use the forces exerted by intensity gradients in the strongly focused beams of light to trap and move microscopic volumes of matter. Other combinations of forces are induced by the interaction between photons, caused by the photon scattering effects. In practice, the field intensity can be adjusted and tuned to produce the desired gradient field and scattering force to produce the suitable trapping force. Hence, the appropriate force can be configured for the transmitter/receiver parts, which can perform long-distance microscopic transportation.

In this proposal, dynamic optical tweezers/vortices are generated using the dark soliton, bright soliton, and Gaussian pulse propagating within an add/drop optical multiplexer incorporated with two nanoring resonators (PANDA ring resonator). The dynamic behavior of soliton and Gaussian pulse is well described by Kachynski et al. ${ }^{23}$ The PANDA ring resonator is a specific device name which is given by the submitted paper authors. The design of original structure of a PANDA ring and its application for drug delivery was reported by Oguchi and Terada. ${ }^{19}$ The parameters of the fabricated device are as close as possible to those of the original design. The references of the practical devices are also given.

In the system proposed in this paper, the blood circulation system and pulmonary network can trap and transport (filter) drug from heart to capillaries. The required trapping tool sizes can be generated and formed for the specific blood circulation with oxygen and finally the clean blood can be sent to the destination via the through port. However, in practice, several sensors are required for environmental and blood quality control, which need to be explored. RBC transport in the capillary network is an indispensible element for this comprehensive model as well as lab-on-a-chip for RBC transport in capillary networks to circulate oxygen and carbon dioxide throughout the human body.,12 This study investigated the use of two different-wavelength tweezers, molecular buffers, and bus networks to form the transported drug volume, especially for delivering and transporting large volumes of drug, suitable for a multi-drug delivery network such as molecular diagnostic networks, blood circulation networks, Alzheimer's and Parkinson's diagnosis, and molecular electronics. In addition, two different-wavelength tweezers were fed into the network to investigate molecular network stability. In practice, the multi-drug delivery networks could be used for large-scale drug delivery.

\section{Principle and method}

In theory, the trapping forces are exerted by the intensity gradients of highly focused light beams to trap and transport the microscopic volumes of matter. The optical forces are customarily defined by the relationship between optical scattering force and gradient force $\left(\mathrm{F}_{\text {grad }}\right){ }^{25}$ Furthermore, in the Rayleigh regime, the trapping forces decompose naturally into two components, since the electromagnetic field is uniform across the dielectric. Thus, the particles can be treated as induced point dipoles. Increasing the numerical aperture (NA) increases the gradient strength due to a decrease in focal spot $\operatorname{size}^{26}$ which can be formed within the tiny system, for instance, a nanoscale device (nanoring resonator). In this proposed system, the trapping force is produced by a dark soliton, in which the valley of the dark soliton is generated and controlled within the PANDA ring resonator by the control port signals. Figure 1 shows the output field $\left(E_{\mathrm{t} 1}\right)$ at the through port. ${ }^{2}$ In the add/drop device, the nonlinear refractive index is ignored because it does not affect the system. The electric fields $E_{0}$ and $E_{0 \mathrm{~L}}$ are the fields circulating within the nanoring at the right and left side of the add/drop optical filter. To form the broad spectrum output, two nonlinear ring resonators are introduced to form a proposed structure called 


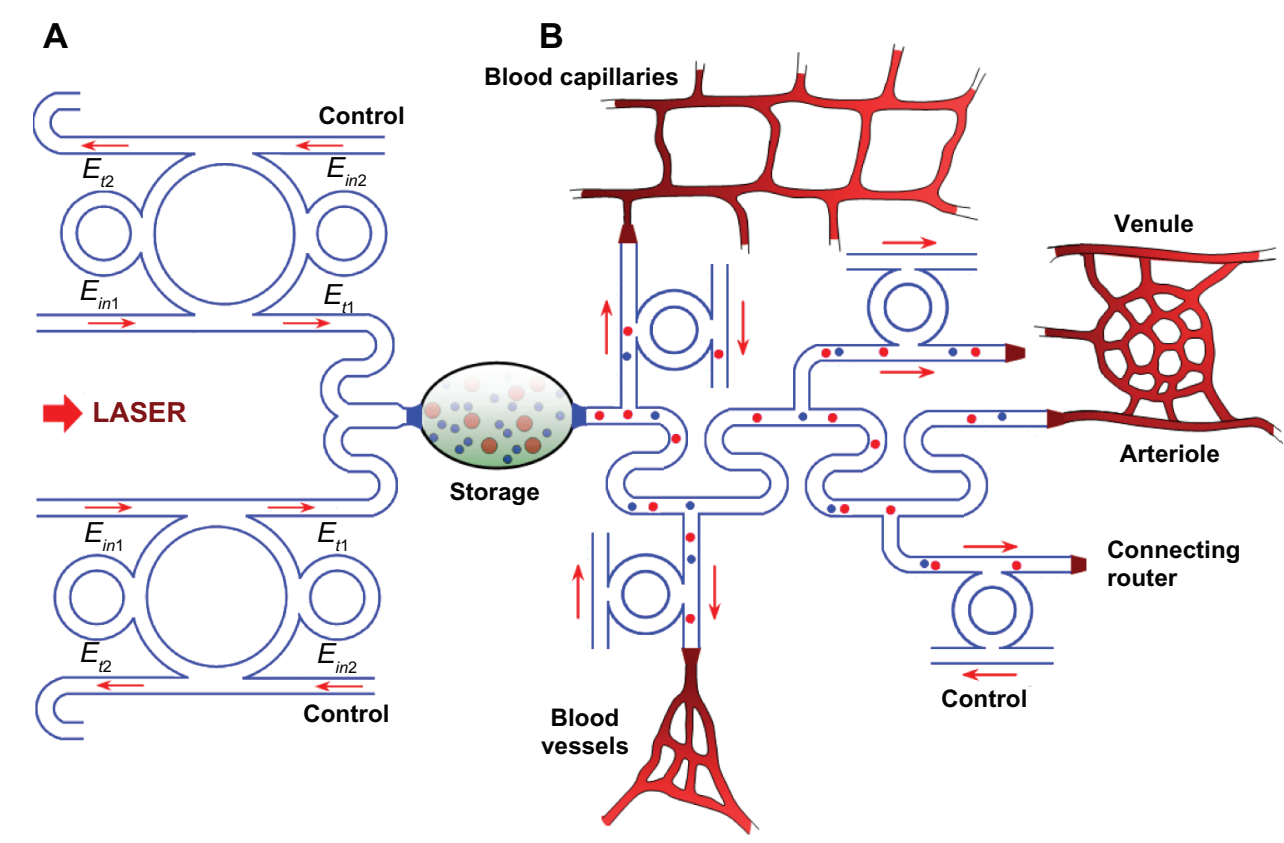

Figure I Schematic diagram of a buffer and bus networks. (A) is a PANDA ring resonator, $(\mathbf{B})$ is a wavelength router and bus capillaries network. $\mathrm{R}_{\text {add }}$ is the add/drop filter radius, $R_{R}$ and $R_{L}$ are the right and left ring resonator radii, respectively.

a PANDA ring. A panda is a well known Chinese bear, which was used to describe the polarization maintaining fiber core structure. In this work, the proposed ring resonator, called a PANDA ring, is a modified add/drop filter. By using the broad-spectrum output, the multitweezers can be generated and molecules can be trapped and transported.

The power output $\left(P_{\mathrm{t} 1}\right)$ at the through port is written as

$$
P_{t 1}=\left|E_{t 1}\right|^{2}
$$

The power output $\left(P_{\mathrm{t} 2}\right)$ at the drop port is

$$
P_{t 2}=\left|E_{t 2}\right|^{2}
$$

\section{Multi-access drug delivery network}

A molecular buffer needs to be included in the system. The molecular buffer plays an important role for storing or delaying atoms/molecules over a period of time, which gives enough time for operation. ${ }^{27,28}$ A molecular buffer is a new device, which is operated in the same way as a gas buffer. ${ }^{29}$ The polarizability of the particle is calculated by Equation (5). In this case, we assume that the spherical particle is polystyrene $(\mathrm{n}=1.5894)$ and the liquid medium is water $(\mathrm{n}=1.33)$. The optical power which is required to trap particles of a certain size/polarizability is $9.1 \mathrm{~W}$ (Figure 2). In simulation, the bright soliton with center wavelength $400 \mathrm{~nm}$, peak power $1 \mathrm{~W}$, and pulse width of 35 fs is fed into the system via the input port, where the coupling coefficients are $\kappa_{0}=0.5, \kappa_{1}=0.35, \kappa_{2}=0.1$, and $\kappa_{3}=0.35$. The ring radii are $R_{\text {add }}=20 \mu \mathrm{m}, \mathrm{R}_{\mathrm{R}}=\mathrm{R}_{\mathrm{L}}=5 \mu \mathrm{m}$. The evidence for the practical device with a radius of $2-3 \mu \mathrm{m}$ has been reported by Zhu et al. ${ }^{30}$ In this case, the dynamic tweezers (gradient fields) are in the form of bright solitons, Gaussian pulses, and dark solitons, which can be used to trap the required microscopic volume. In this investigation four tweezers with different center wavelengths are generated, whose dynamic movements can be seen in Figure 4, where Figure 4A represents tweezers with different sizes and wavelengths and Figure 4B represents tunable tweezers by coupling constant variation. The required drug volumes can be obtained by the drop port outputs.

In practice, the fabrication parameters can be easily controlled by the ring resonator radii instead of coupling constants. The important aspect of this system is that the tunable tweezers can be obtained by tuning (controlling) the add (control) port input signal, in which the required number of microscopic volumes (atom/photon/molecule) can be obtained at the drop/through ports, otherwise, they propagate within a PANDA ring without collapsing/decaying into the waveguide. In practice, the trapped drug molecules can be transported into the wavelength router via the through port, while the retrieved drug volumes are received via the drop port (connecting target). The advantage of the proposed system is that the transmitter and receiver can fabricate onchip and alternatively can be operated by a single device. The magnitude of optical trapping force is in the pico Newton 
A

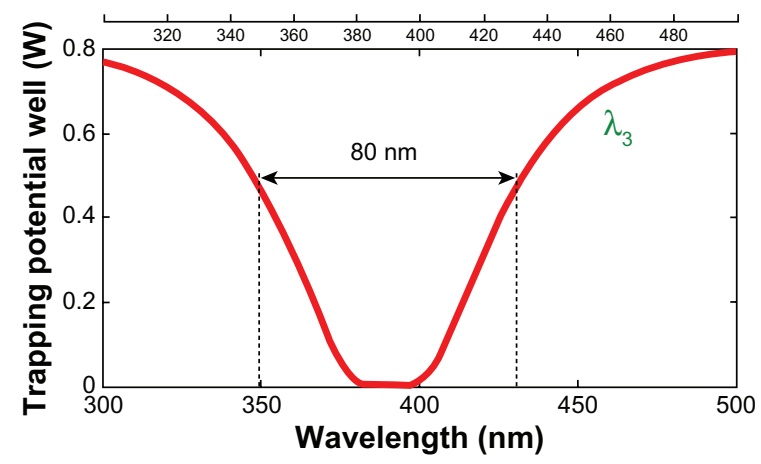

C

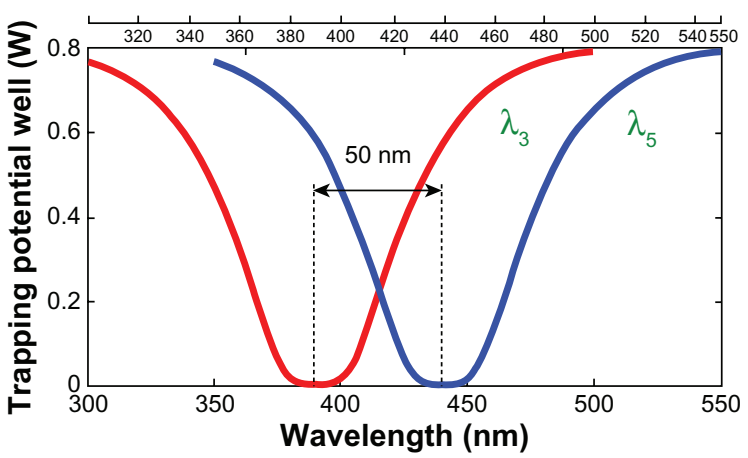

B

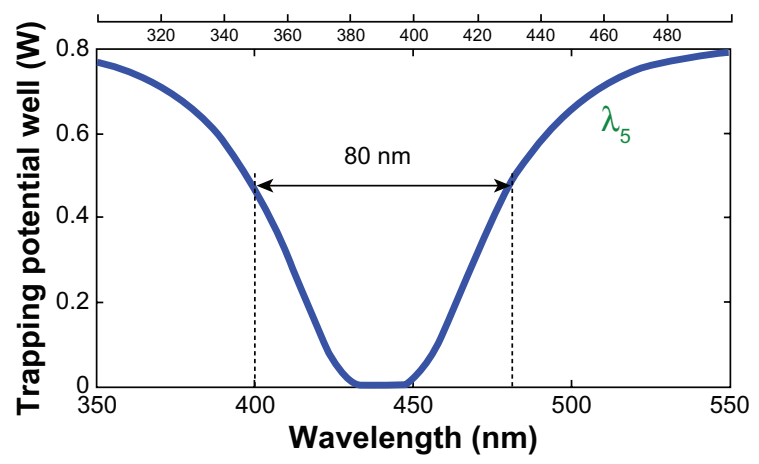

D

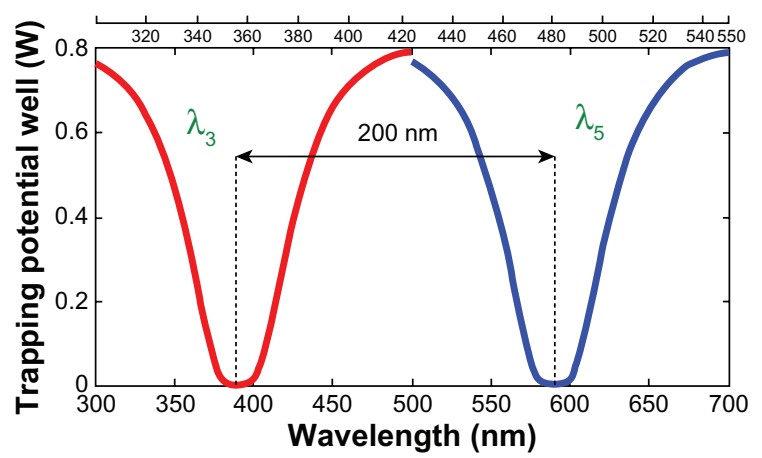

Figure 2 Results of the trapping tools. (A) wavelengths center $400 \mathrm{~nm}$, (B) wavelengths center $450 \mathrm{~nm}$. (C) and (D) are different tweezer separations. $\mathrm{R}_{\text {add }}=2 \mu \mathrm{m}$, $R_{R}=R_{L}=I \mu \mathrm{m}$. The coupling coefficients are $\kappa_{0}=0.5, \kappa_{1}=0.35, \kappa_{2}=0.1$ and $\kappa_{3}=0.35$. The input power is I W.

(pN) range, depending on the relative refractive index of particle. ${ }^{31}$ The particle radius located in the cavity decreases with the decrease in refractive indices compared with the host medium..$^{32,33}$ The waveguide of the drug delivery system can be an optical waveguide with a liquid core which can trap the drug molecules smoothly within the network. By using the drug bus network, the trapped drug molecules can be transported to the required drug targets and the specific drug molecules can be obtained by using the molecular transceiver. To form the trapping tools, the PANDA ring resonator with four ports was used, as shown in Figure 1. First, the dark soliton is fed into the system via the input port. Second, the output trapping tools are transmitted into the throughput port and bus networks. Third, the required drug molecules are filtered and obtained via the drop ports. Finally, molecules are transported within the bus (ring) networks and drug routers, in which the control port is available for additional applications. The molecular trapping probe can be adjusted to select the drug molecule size of $80 \mathrm{~nm}$ or $0.2681 \times 10^{-3} \mu \mathrm{m}^{3}$ per potential well (Figure $3 \mathrm{~A}$ and B), but the fluidics microscopic volume can be transported faster, which depends on the viscosity of media and particle. ${ }^{33}$ This molecular trapping probe can be used for drug molecule transport at the through port and networks, the parameters for which are given in the figure captions (Figure 3). The advantage of the proposed system is that it provides multiple access to the drug volumes and targets. Moreover, the use of mesh networks (combined networks) can also be realized, which can offer a large diagnosis area.

\section{Network stability calculation}

Several reports have shown that fluidics particles (drug volumes) can perform the realistic applications..$^{34,35}$ The system proposed here shows that a tiny device in the form of thin film can be fabricated and used ${ }^{36}$ to integrate drug delivery network into the application area, as shown in Figure 3. Moreover, the use of the proposed system for the blood circulation network of artificial bone is also suitable for in situ surgery and neural and brain diagnosis. By using the design networks, the required trapped volumes can be transported within the network via the molecular buffer (storage) to the required destinations, for instance, the trapped tangle protein can be filtered via the add/drop filter before reaching the desired destinations. The throughput port $\left(E_{\mathrm{t} 1}\right)$ output of add/drop filter is connected to the axon (axon terminal), then to neural cell and dendrite. The effective area of the waveguide is $2.01 \mu \mathrm{m}^{2}$ $(r=800 \mathrm{~nm})$ and the outside diameter of the microtubule is $25 \mathrm{~nm} \cdot{ }^{37}$ Axon diameter at birth is $1 \mu \mathrm{m}$, increasing through 

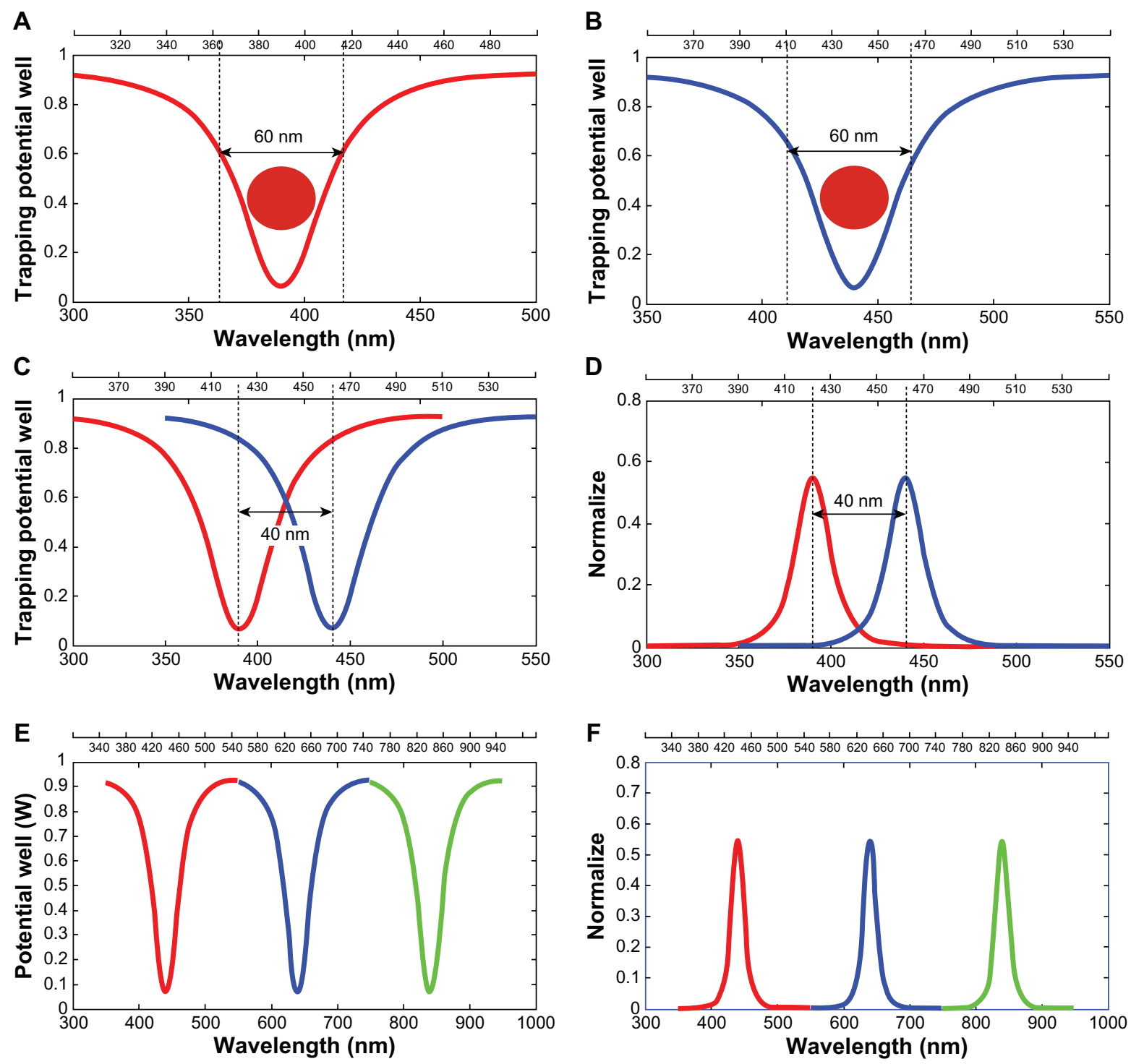

Figure 3 Results of the trapping tools. (A) wavelengths center $400 \mathrm{~nm}$, (B) wavelengths center $450 \mathrm{~nm}$, (C) tweezers separation, (D) normalized tweezers, (E) multitweezers, and $(\mathbf{F})$ normalized tweezers. $R_{\text {add }}=10 \mu \mathrm{m}, R_{R}=R_{L}=3 \mu \mathrm{m}$. The coupling coefficients are $\kappa_{0}=0.95, \kappa_{1}=0.5, \kappa_{2}=0.2$ and $\kappa_{3}=0.5$. The input power is I W.

childhood (7 years) to $12 \mu \mathrm{m}$ and to $24 \mu \mathrm{m}$ at adulthood. ${ }^{38} \mathrm{In}$ Alzheimer's diagnosis ${ }^{39}$ the optical tool is connected between the axon and the nerve cells and can be used to trap the tangle protein into the removal storage by add/drop filter (control port). The bus network design can also be used to trap the molecular motor to activate the information of neuronal cell at the same time. For better access, the coupling material is required to use as waveguide-axon coupling.

In operation, as "networks" are made up of add/drop filters (Figure 4), their performance depends on the add/drop filters. Micro- and nanowaveguides are gaining prominence in this field. Filters offer good stability and isolation between channels at moderate cost. The add/drop filters' capability affects the network size. The maximum nodes of a network depend on the maximum amount of channels of add/drop filter. The popular dense wavelength division multiplexing (DWDM) component with many channels has been achieved in both laboratory and theoretical works. ${ }^{40,41}$ This means that multi-variable routers or networks with many ports can be built in future.

In this work, we propose the use of an optical network principle to estimate network stability. In general, the problems of a large network having the stability of a small network are insertion loss (IL) and crosstalk effect (FC). The IL reduces the efficient transmission distance. In popular communication networks, in which the signals pass through the router, insertion loss is usually $5 \mathrm{~dB}$. According to the performance of present point-to-point transmission systems, we can build a required network over at least $50 \mathrm{~km}$. Along with the development of DWDM technology, the insertion 

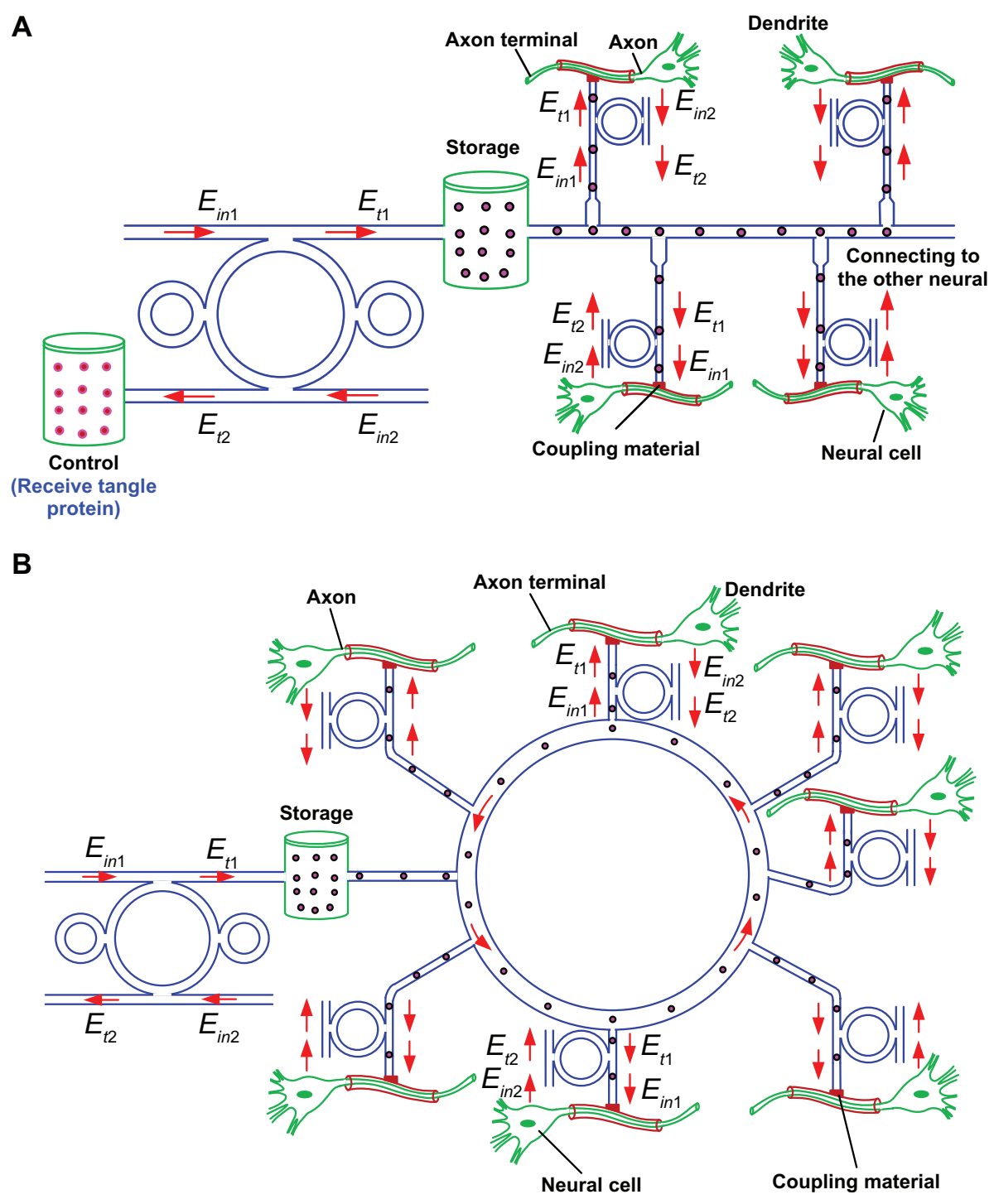

Figure 4 Schematic diagram of molecular network (A) bus network (B) ring network.

loss can be reduced to less than $1 \mathrm{~dB}$ in future. ${ }^{42}$ Then the multivariable network will cover more than $100 \mathrm{~km}$ with high capacity. The crosstalk effect is mainly due to a signal of co-channel interference and adjacent-channel interference. The crosstalk can be considered in terms of channel separability. For a network, crosstalk brings bit errors, so it must be reduced as low as possible. $I L$ and $F C$ can be estimated as:

$$
\begin{gathered}
I L=10 \times \log \left(\frac{P_{\text {in }}}{P_{\text {out }}}\right) \\
F C_{j}\left(\lambda_{i}\right)=10 \times \log \left[\frac{P_{j}\left(\lambda_{i}\right)}{P_{i}\left(\lambda_{i}\right)}\right]
\end{gathered}
$$

Here $P_{\text {in }}$ and $P_{\text {out }}$ are input and output of soliton, $P_{j}\left(\lambda_{i}\right)$ is output of soliton with wavelength $\lambda_{i}$ which exports from port $j$,
$P_{i}\left(\lambda_{i}\right)$ is output of soliton with wavelength $\lambda_{i}$ which exports from port i. $P_{i}(\lambda)$ in Equation (4) is equal to $P_{\text {out }}$ in Equation (3).

$$
\begin{gathered}
\frac{P_{i}\left(\lambda_{i}\right)}{P_{\text {in }}}=\frac{P_{i}\left(\lambda_{i}\right)}{P_{\text {out }}} \times \frac{P_{\text {out }}}{P_{\text {in }}}=10 \frac{\left[F C _ { j } \left(\lambda_{i)-L L]}\right.\right.}{10} \\
P_{\text {out }} / P_{\text {in }}=10^{-I L / 10}
\end{gathered}
$$

We first assume that all input photons from any user are the same when they enter the router. Since one soliton can pass through two add/drop filters when it passes a router, the crosstalk versus efficient signals is given as:

$$
\left[\frac{P_{i}\left(\lambda_{i}\right)}{P_{\text {in }}}\right]^{2} \times\left[\frac{P_{\text {in }}}{P_{\text {out }}}\right]^{2}=10^{\frac{2 \times F C_{j}(\lambda i)}{10}}
$$


Now we consider the situation in which the input soliton is not the same. A bad situation is that input photons which produce efficient signals pass through a device that has $\mathrm{X}$ $\mathrm{dB}$ insertion loss before passing through the router but those solitons which produce crosstalk do not. The ratio in Equation (7) will become $10^{[X+2 \times F C j(\lambda i)] / 10}$. If there are many inputs that produce crosstalk, the ratio must be

$$
\sum_{j=1}^{N-1} 10 \frac{\left[X+2 \times F C j\left(\lambda_{1}\right)\right]}{10}
$$

Here $j \neq i$, where $\mathrm{N}$ is the number of channels (receiver nodes).

In present study, the system dimensions have been reduced to be micro-/nanoscale. From Figure 4, the coupling ratio of each coupling point is $50: 50$, ie, $3 \mathrm{~dB}$ coupling power. The molecular bus network has $\mathrm{N}=4, F C_{j}\left(\lambda_{\mathrm{i}}\right)<-4.26 \mathrm{~dB}$ (when $j=i \pm 1$ ), $X=-0.97 \mathrm{~dB}$. The normalized input is 0.8 ; $\lambda_{i}$ and $\lambda_{j}$ equal 400 and $450 \mathrm{~nm}$, respectively. Their ratio is less than $0.113 \%$. Therefore the errors resulting from crosstalk are less than $1(10 \%)$ and can be ignored. Along with the development of DWDM technology, crosstalk will be smaller and the performance of the molecular router or network will improve. Thus, we can easily build a feasible multi-variable network for multi-drug delivery applications, ie, for large networks.

\section{Conclusions}

We have proposed an interesting system that can be used for multi-drug delivery networks. The trapped drug molecules can move into the liquid core waveguide and networks by using optical tweezers, in which drug can be trapped, stored, and delivered via the molecular network. Such a system can also be used for large-scale molecular drug delivery network and diagnosis. The mesoscopic particle can be trapped and transported within the waveguide and network such as nanocarrier (polymeric nanoparticles, dendrimers) and lipid-based drug carriers. ${ }^{43,44}$ By using practical device parameters, such a proposed system can be fabricated and integrated into a practical thin film device. The trapping and movement of molecules in the system can be used for certain diagnostic purposes and to deliver small molecules to their target organ in the human body. Network stability was also calculated and it was found that the crosstalk effects due to the two wavelength-trapping drug molecules can be ignored. The proposed system can be used for long-distance transport of drug molecules in multidrug delivery networks, in which drug delivery or molecular communication can be performed via the wavelength router and bus network, which will be available for large network systems (neural systems) in the near future. The proposed technique can be used in the new era of electronics and communications, where the use of molecules, DNA, genes, and atoms can have various applications within a tiny system.

\section{Acknowledgments}

We would like to thank the Institute of Advanced Photonics Science, Nanotechnology Research Alliance, Universiti Teknologi Malaysia (UTM) and King Mongkut's Institute of Technology (KMITL), Thailand for providing the research facilities. This research work has been supported by UTM's Tier 1/Flagship Research Grant, MyBrain15 Fellowship/ MOHE SLAB Fellowship and the Ministry of Higher Education (MOHE) research grant. N. Suwanpayak would like to acknowledge King Mongkut's Institute of Technology Ladkrabang, Bangkok (KMITL), Thailand for the partial support in higher education at KMITL, Thailand.

\section{Disclosure}

No conflicts of interest were declared in relation to this paper.

\section{References}

1. Scanlon VC. Essentials of Anatomy and Physiology. 5th ed. Riverdale, New York: College of Mount St Vincent; 2007.

2. Li Y, Wen C, Xie H, Ye A, Yin Y. Mechanical property analysis of stored red blood cell using optical tweezers. Colloids Surf B Biointerfaces. 2009:70;169-173.

3. Obrist D, Weber B, Buck A, Jenny P. Red blood cell distribution in simplified capillary networks. Phil Trans R Soc A. 2010;368:2897-2918.

4. Fontes A, Barjas Castro ML, Brand MM, et al. Mechanical and electrical properties of red blood cells using optical tweezers. $J$ Opt. 2011;13:044012.

5. Lee K, Kim C, Jung G, Kim TS, Kang JY, Oh KW. Microfluidic network-based combinatorial dilution device for high throughput screening and optimization. Microfluid Nanofluid. 2010;8:677-685.

6. Ashkin A, Dziedzic JM, Yamane T. Observation of a single-beam gradient force optical trap for dielectric. Opt Lett. 1986;11:288-290.

7. Feng Q, Park JS, Vida L, et al. Biological properties and enucleation of red blood cells from human embryonic stem cells. Blood. 2008;112:4362-4363

8. Chen HD, Ge K, Li Y, et al. Application of optical tweezers in the rresearch of molecular interaction between lymphocyte function associated antigen-1 and its monoclonal antibody. Cell Mol Immunol. 2007;4:221-225.

9. Ashkin A, Dziedzic JM. Optical trapping and manipulation of viruses and bacteria. Science. 1987;235:1517-1520.

10. Zhao X, Sun Y, Bu J, Zhu S, Yuan XC. Microlens array enabled on-chip optical trapping and sorting. Applied Opt. 2011;50:318-322.

11. Lee WG, Park K, Bang H, et al. Single red blood cell defromnility test using optical trapping in plastic microfluid chip. Proceedings of the 31 Annual International IEEE EMBS Special Topic on Conference Microtechnologies in Medicine and Biology, Kahuku, Oahu, Hawaii; May 12-15, 2005:389-390.

12. Chen YC, Chen GY, Lin YC, Wang GJ. A lab-on-a-chip capillary network for red blood cell hydrodynamics. Microfluid Nanofluid. 2010;9:585-591. 
13. Suwanpayak N, Jalil MA, Teeka C, Ali J, Yupapin PP. Optical vortices generated by a PANDA ring resonator for drug trapping and delivery applications. Bio Med Opt Express. 2011;2:159-168.

14. Cheng M, Wu C, Hiltunen J, Wang Y, Wang Q, Myllylä R. A variable delay optical buffer based on nonlinear polarization rotation in semiconductor optical amplifier. IEEE Photon Technol Lett. 2009;21:1885-1887.

15. Liu J, Lee TT, Jiang X, Horiguchi S. Blocking and delay analysis of single wavelength optical buffer with general packet size distribution. J Lightwave Technol. 2009;27:955-966.

17. Dragone CP. Improved waveguide grating optical router suitable for CWDM. EP 2250523 (patent). 2010.

18. Ham BS. Delayed optical router/switch. US 20100232792 (patent). 2010.

19. Oguchi K, Terada S. Optical network system, optical router, fault recovery method of optical network, and program. JP 2010063009 (patent). 2010.

20. Piyatamrong B, Kulsirirat K, Mitatha S, Yupapin PP. Dynamic potential well generation and control using double resonators incorporating in an add/drop filter. Mod Phys Lett B. 2010;24:3071-3082.

21. Cai H, Poon A. Optical manipulation and transport of microparticle on silicon nitride microring resonator-based add-drop devices. Opt Lett. 2010;35:2855-2857.

22. Egashira K, Terasaki A, Kondow T. Photon-trap spectroscopy applied to molecules adsorbed on a solid surface: Probing with a standing wave versus a propagating wave. App Opt. 1998;80:5113-5115.

23. Kachynski AV, Kuzmin AN, Pudavar HE, Kaputa DS, Cartwright AN, Prasad PN. Measurement of optical trapping forces by use of the two-photon-excited fluorescence of microspheres. Opt Lett. 2003;28:2288-2290.

24. Schulz M, Crepaz H, Schmidt-Kaler F, Eschner J, Blatt R. Transfer of trapped atoms between two optical tweezer potentials. J Mod Opt. 2007;54:1619-1626.

25. Svoboda K, Block SM. Biological applications of optical forces. Ann Rev Biophy Biomol Struct. 1994;23:247-282.

26. Zhu J, Ozdemir SK, Xiao YF, Li L, He L, Chen DR. On-chip single nanoparticle detection and sizing by mode splitting in an ultrahigh- $Q$ microresonator. Nat Photonics. 2010;4:46-49.

27. Rosenberry MA, Reyes JP, Tupa D, Gay TJ. Radiation trapping in rubidium optical pumping at low buffer-gas pressures. Phys Rev A. 2007;75:023401-1-6.

28. Lignie MC, Woerdman JP. Light-induced drift of $\mathrm{Na}$ in molecular buffer gases. J Phys B: At Mo Opt Phys. 1990;23:417-426.
29. Waggoner PS, Palmer JS, Antonov VN, Weaver JH. Metal nanostructure growth on molecular buffer layers of $\mathrm{CO}_{2}$. Surf Sci. 2005;596:12-20.

30. Zhu J, Ozdemir SK, Xiao YF, Li L, He L, Chen DR. On-chip single nanoparticle detection and sizing by mode splitting in an ultrahigh- $Q$ microresonator. Nat Photonics. 2010;4:46-49.

31. Fischer M, Sørensen KB. Calibration of trapping force and response function of optical tweezers in viscoelastic. J Opt A Pure App Opt. 2007;79:239-250

32. Nieminen TA, Dunlop HR, Heckenberg NR. Calculation and optical measurement of laser trapping forces on non-spherical particles. J Quantitative Spectro and Rad Trans. 2001;70:627-637.

33. Segev M, Christodoulides DN, Rotschild C. Method and system for manipulating fluid medium. US 2011/0023973 A. (patent) 2011.

34. Erikssonv E, Enger J, Nordlander B, et al. A microfluidic system in combination with optical tweezers for analyzing rapid and reversible cytological alterations in single cells upon environmental changes. $L a b$ Chip. 2007;7:71-76.

35. Chen SY, Hu SH, Liu DM, Kuo KT. Drug delivery nanodevice, its preparation method and used thereof. US 2011/0014296 A1 (patent). 2011.

36. Macleod HA. Thin-Film Optical Filter. 4th ed. New York: Taylor and Francis; 2010

37. Karp G. Cell and Molecular Biology. 6th ed. Hoboken, NJ: John Wiley and Sons, Inc; 2010.

38. Paus T, Toro R. Could sex differences in white matter be explained by g ratio? Neuro. 2009;3:1-7.

39. Mitatha S, Moongfangklang N, Jalil MA, et al. Proposal for Alzheimer's diagnosis using molecular buffer and bus network. Int J Nanomedicine. 2011;6:1209-1216.

40. Takada K, Abe M, Shibata T, Okamoto K. Field demonstration of over 1000-channel DWDM transmission with supercontinuum multi-carrier source. Electron Lett. 2002;38:572-573.

41. Sarapat K, Ali J, Yupapin PP. A novel storage and tunable light source generated by a soliton pulse in a micro ring resonator system for super dense wavelength division multiplexing use. Microw Opt Technol Lett. 2009;51:2948-2952.

42. Smit MK. Progress in AWG design and technology. Proceedings of WFOP 2005. 4th IEEE/LEOS workshop on fibres and optical passive component; 2005:26.

43. Cho K,Wang X, Nie S, Chen Z, Shin DM. Therapeutic nanoparticles for drug delivery in cancer. Clin Cancer Res. 2008;14:1310-1316.

44. Ravi Kumar MNV. Nano and microparticles as controlled drug delivery devices. J Pharm Pharmaceut Sci. 2000;3:234-258.
International Journal of Nanomedicine

\section{Publish your work in this journal}

The International Journal of Nanomedicine is an international, peerreviewed journal focusing on the application of nanotechnology in diagnostics, therapeutics, and drug delivery systems throughout the biomedical field. This journal is indexed on PubMed Central, MedLine, CAS, SciSearch $\AA$, Current Contents ${ }^{\circledR} /$ Clinical Medicine,

\section{Dovepress}

Journal Citation Reports/Science Edition, EMBase, Scopus and the Elsevier Bibliographic databases. The manuscript management system is completely online and includes a very quick and fair peer-review system, which is all easy to use. Visit http://www.dovepress.com/ testimonials.php to read real quotes from published authors. 